\title{
Factores que condicionan la alimentación de personas adultas en un centro de rehabilitación de drogas
}

\author{
Claudia Troncoso Pantoja ${ }^{1}$, Darlyng Mora Ormeño ${ }^{2}$, Paulina Sánchez Espinoza ${ }^{2}$
}

\section{RESUMEN}

Objetivo: Interpretar la percepción de las condicionantes que influyen en la alimentación de un grupo de usuarios internos en centro de rehabilitación para drogas de la comuna de Concepción, Chile, durante el año 2015.

Material y métodos: A través de un diseño cualitativo con enfoque fenomenológico, se entrevistó internos de centro de rehabilitación para drogas, que cumplían con criterios de selección asignados para el estudio. La obtención de información se realizó a través de entrevistas semiestructuradas, que fueron validadas por expertos y sometida a prueba piloto. El muestreo fue no probabilístico por conveniencia mediante caso tipo. El tamaño muestral se obtuvo con punto de saturación teórico, se completó en la octava entrevista. Los resultados fueron sometidos a triangulación de informantes y analizados utilizando la técnica de análisis de discurso.

Resultados: Los participantes identifican factores positivos y negativos que condicionan su alimentación. Reconocen como positivo el presentar tiempos y horarios de comidas establecidos, que les ayuda a ordenar más su alimentación, sin embargo, situaciones de estrés dentro del recinto son descritas como limitantes en su estado anímico, afectando su apetito y reduciendo su ingesta alimentaria. Además, no estar con sus familias, en especial al inicio del tratamiento, también es considerado como un aspecto negativo, lo mismo que la limitación en la variedad de preparaciones ofertadas en el centro, ya que no responden a sus preferencias alimentarias. Durante su etapa de abstinencia, incrementan el consumo de alimentos aportadores de carbohidratos de absorción rápida.

Conclusiones: Se interpreta que los entrevistados en el centro de rehabilitación de drogas identifican condicionantes que influyen en su alimentación, los que actúan favoreciendo o limitando el consumo de alimentos y la relación que presentan estas personas frente a la alimentación que reciben mientras están en etapa de abstinencia.

Palabras clave: drogas, rehabilitación, alimentación, estudio cualitativo.

\section{Factors affecting adult feeding in a Drug rehabilitation Center}

\section{ABSTRACT}

Objective: To interpret the perception of the determining factors that influence the diet of a group of intern patients in a drug rehabilitation center in Concepción, Chile, during the year 2015.

Materials and methods: Through a qualitative design with a phenomenological approach, intern patients of a drug rehabilitation facility, who fulfilled the study assigned criteria, were interviewed. The collection of data was conducted through semi structured interviews; which were validated by experts and subject to a pilot test. Nonprobability convenience sampling was performed. The sample size was determined through theoretical saturation, which was completed at the eighth interview. The results were subject to triangulation of informants, and were analyzed using the technique of discourse analysis.

Results: The participants identify positive and negative factors that condition their diet. They claim that having a fixed schedule is positive since this helps them to have a more organized diet. However, some stressful situations within the facilities are described as limiting factors in their mood, thus affecting their appetite and reducing their food intake. Additionally, not being with their families, especially at the beginning of the treatment, is considered a negative aspect. The lack of variety in terms of food preparation is also negative since this does not correspond to their food preferences. During the drug withdrawal stage, the intake of food containing carbohydrates of rapid absorption was increased.

Conclusions: It is interpreted that the interviewees at the drug rehabilitation facility identify conditioning factors that influence their diet, which promote or inhibit food intake, and affect the attitude they present towards their diet in the drug withdrawal stage.

Key words: drugs, rehabilitation, diet, qualitative study.

1. Magister en Salud Pública Basada en Evidencias. Facultad de Medicina. Departamento de Ciencias Clínicas y Preclínicas. Carrera de Nutrición y Dietética. Universidad Católica de la Santísima Concepción. Chile.

2. Licenciada en Nutrición y Dietética. Facultad de Medicina. Carrera de Nutrición y Dietética. Universidad Católica de la Santísima Concepción. Chile. 


\section{INTRODUCCIÓN}

Las drogas y su uso afectan directamente, así como indirectamente, a la sociedad, las familias y los propios individuos, con costos no solo económicos, también emocionales y de calidad de vida para estas personas, por lo que es considerado un problema social y de salud pública que aqueja a distintos grupos etarios $^{(1,2,3)}$.

A nivel mundial, se estima que los consumidores de sustancias ilícitas alcanzan cifras por sobre los 208 millones. Se identifica que las drogas son causantes del $50 \%$ de los arrestos policiales, muertes por accidentes y homicidios; $30 \%$ de los suicidios y $70 \%$ de los casos de violencia intrafamiliar. La Organización Panamericana de Salud (OPS) reconoce tres factores que condicionan los problemas de drogadicción en América Latina: desigualdad en el desarrollo; falta de acceso a servicios de salud y la exclusión social de ciertos sectores de la población ${ }^{(4,5,6)}$.

Las drogas se clasifican en aquellas que producen estimulación o depresión del Sistema Nervioso Central (SNC), como el alcohol, hipnóticos, sedantes u opiáceos, cocaína o anfetaminas. Las de mayor consumo son las conocidas como lícitas, en la que se destaca el alcohol y tabaco. En estos últimos años aumentó el uso de sustancias ilícitas como cannabis, cocaína, éxtasis, alucinógenos y sustancias volátiles ${ }^{(7,8)}$. Estas últimas actúan alterando el funcionamiento del SNC y el comportamiento de quien las utiliza, además del estado de conciencia, humor y su cognición. Estas sustancias adictivas pueden producir otras alteraciones como intoxicación y/o abstinencia ${ }^{(9,10)}$.

El uso y abuso de drogas puede ser prevenido, sin embargo, la dependencia a estas sustancias debe ser tratada. El tratamiento de la dependencia presenta un enfoque biopsicosocial, con terapias psicológicas y/o farmacológicas que, en casos más serios, deben realizarse en centros de rehabilitación de atención cerrada, con resultados no siempre satisfactorios para la persona atendida. La Organización Mundial de la Salud (OMS) identifica que el tratamiento se inicia cuando el consumidor de sustancias psicoactivas se contacta con un proveedor de salud (o de otro servicio comunitario) y a través de diversas intervenciones, alcanzan un adecuado nivel de salud y bienestar ${ }^{(11-15)}$.

En general, estos centros de rehabilitación prestan atención, tratamiento y rehabilitación a los usuarios atendidos, con recursos humanos, materiales y físicos adaptados para las actividades y funciones a realizar, aunque un número limitado de personas se atiende en estos centros, debido entre otros factores a que el entorno y el mismo individuo no perciben como el consumo como problemático o que esté fuera de control $^{(16,17)}$.

En Chile, las políticas destinadas a la atención de estas personas en centros cerrados consideran la necesidad de presentar comedores que atiendan al $50 \%$ de los usuarios en forma simultánea y caracterización de la planta física para el almacenamiento, manipulación y entrega de las preparaciones culinarias, sin profundizar en los aspectos nutricionales que estas últimas deben presentar $^{(18)}$.

En base a la importancia que presenta, entre otros aspectos, una adecuada alimentación y nutrición en el tratamiento de rehabilitación de personas con drogodependencia, surge esta investigación que presenta como objetivo el interpretar la percepción de las condicionantes que influyen en la alimentación de un grupo de usuarios internos en centro de rehabilitación para drogas perteneciente a la comuna de Concepción, Chile, durante el año 2015.

\section{MATERIAL Y MÉTODOS}

Para profundizar en la experiencia que presenta la alimentación para un grupo de adultos en etapa de rehabilitación de drogas se utiliza como diseño metodológico el paradigma cualitativo con enfoque fenomenológico.

Los participantes en el estudio fueron internos adultos de sexo masculino en situación de pobreza y con necesidades de apoyo en centro de rehabilitación para consumo problemático de drogas ilícitas, especialmente pasta base de cocaína, ubicado en la provincia de Concepción, región del Bíobio, Chile. Se debe destacar que mujeres del centro de rehabilitación, fueron invitadas a participar voluntariamente en el estudio, situación que no se pudo concretar, debido a la falta de disposición de estas internas.

El muestreo utilizado fue de tipo no probabilístico por conveniencia, respondiendo a los criterios de selección de la muestra: hombres y mujeres con internación cerrada en Programa de Rehabilitación de Drogas de a lo menos tres meses, con adecuado comportamiento dentro del centro y que firmaran el consentimiento informado. 
Factores que condicionan la alimentación de personas adultas en un centro de rehabilitación de drogas

La muestra se obtiene del punto de saturación teórico, es decir, al no encontrar nuevos datos que aporten al objetivo de la investigación ${ }^{(19)}$. Esta saturación se logró en la entrevista número ocho.

Como instrumento de recolección de datos, se utilizó una entrevista semiestructurada, la que fue sometida a validación por expertos y prueba piloto. La entrevista fue realizada en forma individual en condiciones estándar, dentro del centro de rehabilitación, en acuerdo con la Dirección del Centro y la aprobación voluntaria de los participantes.

La entrevista fue grabada en dispositivo de audio y los resultados traspasados a formato papel por investigadores para ser analizada. Para esta última acción, se utilizó un análisis de contenido, en donde se identificaron los respectivos códigos de base que permitieron obtener los resultados del estudio. Para fortalecer la validez de los resultados, se utilizó una triangulación de investigadores.

\section{Aspectos éticos}

El estudio consideró para los aspectos éticos la firma del consentimiento informado, en donde se comunicó al entrevistado sobre los objetivos de la investigación, la metodología de la recolección de la información y su posterior análisis, garantizando la privacidad de la persona que participó en forma voluntaria, omitiendo el nombre y los antecedentes personales al momento de la entrevista y en el desarrollo del informe de la investigación. A lo anterior, antes de iniciar la recolección de la información, se entrevistó y solicitó autorización a las autoridades del centro de rehabilitación, profundizando en los objetivos del estudio y metodología de obtención de la información.

\section{RESULTADOS}

Percepción de condicionantes que obstaculizan la alimentación en la etapa de rehabilitación de drogas

Variadas son las condicionantes percibidas por los entrevistados que afectan negativamente en su alimentación. Una de ellas son las diferencias de opinión que se puede presentar entre internos, ya sean físicas $y / o$ verbales. Esto provoca una modificación en su estado anímico, generando en ellos un descontento, el que finalmente es percibido como una condicionante en la disminución de su apetito. Esto último, no necesariamente se da si ellos son participes de las discusiones, sino que también se ven afectados si son observadores de estas.

El entorno familiar, referido al no estar físicamente con ellos, también es reconocido como una condicionante en la alimentación de estas personas, ya que esta carencia afectiva influye directamente en la disminución del apetito, especialmente en los primeros días que ingresan al programa de rehabilitación, en contraste con los usuarios que no tienen un hogar constituido, los que declaran que los primeros días en el centro terapéutico sus deseos de alimentarse aumentan considerablemente, atribuyendo directamente esta condición al periodo de abstinencia que recién comenzaban.

Por otra parte algunos usuarios mencionan que al momento de internarse, se produce sintomatología gastrointestinal como la presencia de reflujo o pirosis, lo que influye negativamente en su deseo de alimentarse. Sin embargo, refieren que esta situación no limita totalmente el consumo de alimentos, ya que como están en un régimen cerrado en donde se presentan horarios establecidos para los tiempos de comidas, se alimentan, ya que no existirá otro horario para regularizar su apetito.

Las preparaciones en que son entregados los alimentos en el centro de rehabilitación también son consideradas como condicionantes de la alimentación: aquellas que no están presente entre las preferencias alimentarias de los entrevistados, no son ingeridas, limitando su consumo alimentario.

Otra limitante identificada por los entrevistados para su alimentación, es la escasa variedad de preparaciones que presenta el centro de rehabilitación. Parte importante de los entrevistados mencionan que si existiera más de una opción en tiempos de comidas como el almuerzo y cena, esto influiría positivamente en sus deseos de alimentarse, lo que a su vez es percibido como un refuerzo positivo en el proceso de rehabilitación. También aluden que hasta el estado de ánimo se modificaría positivamente, ya que cuando existen alimentos o preparaciones que no son de su agrado no los consumen, deteriorando su estado anímico.

Percepción de condicionantes que favorecen la alimentación en la etapa de rehabilitación de drogas

Para la gran mayoría de los participantes, se presentan diferencias entre la alimentación recibida 
en el centro de rehabilitación de drogas y su hogar, percibiendo un número importante de entrevistados factores positivos en el centro de atención. En esta comparación destacan múltiples diferencias, como por ejemplo, que en su hogar no se alimentan en forma adecuada, en cantidad y calidad. En cambio declaran que la alimentación entregada por el centro de rehabilitación es recibida todos los días, con adecuados tiempos de comidas y en sus respectivos horarios.

Para la mayoría de los entrevistados, los tiempos de alimentación y horarios de comidas establecidos son parte fundamental de su proceso de rehabilitación, ya que se regularizan conductas alimentarias. Aunque algunos mencionan que las porciones que se les otorgan son reducidas, señalan que son las adecuadas para mantener el estado nutricional normal.

Percepción del consumo de alimentos en período de abstinencia a drogas

Los internos perciben que en general, todos los alimentos son tolerados en forma adecuada en el periodo de abstinencia a las drogas. Solo algunos de los entrevistados mencionan que los alimentos ricos en carbohidratos ya sean complejos y especialmente simples, son muy bien tolerados al comienzo de este periodo de abstinencia, por el hecho de que sienten una mayor sensación de saciedad al ingerirlos. Los deseos de consumir estos alimentos ricos en carbohidratos las primeras semanas de estadía de los usuarios, es interpretado como desencadenante en el aumento de peso drástico que sufrieron algunos de los entrevistados.

Los deseos de alimentarse en los usuarios del programa terapéutico, son percibidos como muy relacionado a la necesidad de consumir droga; se puede interpretar que la falta de estas últimas la sustituyen por alimentos.

\section{DISCUSIÓN}

En general, las investigaciones desarrolladas en personas internadas en centros o programas con dependencia a sustancias ilícitas, se concentran en la importancia que presenta para los usuarios el plan de tratamiento y rehabilitación de drogas, destinado por ejemplo, a mejorar su relación familiar o estilo de vida a través de una atención integral e integrada, que facilite su recuperación ${ }^{(20,21,22)}$, desplazando la importancia de la nutrición y alimentación para estas personas mientras están en tratamiento.
En nuestro estudio, los usuarios relacionaban directamente su estado de ánimo con la ingesta de alimentos mientras estaban internados. Esta situación es identificada en jóvenes que consumen drogas, presentando alteraciones en su estado anímico, principalmente depresión ${ }^{(23)}$ y existiendo una relación directa entre el consumo de alimentos y el estado anímico ${ }^{(24)}$. En el trabajo realizado por Mené L. ${ }^{(25)}$ se interpretó las necesidades nutricionales que presentaron un grupo de hombres de una Comunidad Terapéutica en España, concluyendo que el estado de ánimo condicionaba directamente su consumo alimentario, ya que este último influía directamente al momento de alimentarse, reduciendo su ingesta alimentario o por el contrario, aumentándola notablemente.

Diversas investigaciones profundizan en la importancia que presenta la familia en la persona que está en el centro de rehabilitación ${ }^{(26,27,28)}$. En nuestro estudio, los entrevistados que presentan una estructura de familia regular, también perciben esta importancia, en especial al ingresar al centro, ya que relacionan una disminución en su consumo de alimentos, situación que no es percibida en los pacientes que no identifican un mayor apoyo familiar en su tratamiento.

En cuanto a la alimentación entregada en el centro cerrado de atención, los entrevistados mencionan que algunas de las preparaciones no están entre sus preferencia alimentarias, por lo tanto, no son consumidas, condicionando negativamente en su percepción alimentaria. Las centrales de producción deben ofrecer menús que aseguren su consumo para que de esta manera cubran las necesidades nutritivas de la persona que se encuentran en un determinado lugar en el momento de comer sin presentar la opción de desplazarse, sin embargo, en población internada en distintos centros o en población ambulatoria, la alimentación entregada por centros de alimentación colectiva no son bien evaluados ${ }^{(29-32)}$. Esta situación se replica en los resultados de nuestro estudio, sin embargo, es importante señalar que la percepción negativa se refiere a las preparaciones entregadas en el centro, ya que refieren como fortaleza el recibir alimentación en distintos tiempos y horarios de alimentación, situación que no se presentaba en sus hogares y que es interpretado como positivo para su calidad de vida.

Para los períodos de abstinencia de drogas duras, se producen alteraciones en el tracto digestivo y en el comportamiento alimentario, reflejado en una 
Factores que condicionan la alimentación de personas adultas en un centro de rehabilitación de drogas

inadecuada alimentación ${ }^{(33,34)}$. En nuestro estudio, se cumple esta premisa, ya que los entrevistados mencionan alteraciones en su apetito, con un aumento en el consumo de alimentos aportadores de carbohidratos de absorción rápida, lo que favorecería el incremento de peso en estas personas, afectando su calidad de vida.

En conclusión, se interpreta que los entrevistados en el centro de internación para rehabilitación de drogas identifican condicionantes que influyen en su alimentación, como la familia, estado anímico, situaciones de estrés o preferencias alimentarias, las que actúan favoreciendo o limitando el consumo de alimentos y la relación de estas personas frente a la alimentación recibida.

Independiente del grupo etario o la situación de salud que la persona presenta y que justifica su internación en cualquier centro de atención cerrada, el fin último de todos los agentes sanitarios es procurar mejorar o mantener una adecuada calidad de vida en el usuario atendido. Esta premisa se extrapola a las personas que se encuentren internadas para el control de sus adicciones. Si bien el fin último de los establecimientos es controlar su motivo de ingreso, el considerar en forma más holística su tratamiento, incorporando entre otras aristas la alimentación, en especial las preferencias alimentarias de los internos, sería un factor que no solo favorecería la nutrición de estas personas, sino también, mejoraría su calidad de vida, ya que una adecuada alimentación, no es solo la entrega de nutrientes, también existe un aspecto sociocultural que es necesario para su reinserción.

Entre las limitaciones de este estudio, se encuentra la participación solo de internos de sexo masculino, debido a la negación por parte de las mujeres residentes, por lo que sería necesario próximas investigaciones que refuercen la percepción de las usuarias de estos centros frente a la temática de su alimentación.

\section{REFERENCIAS BIBLIOGRÁFICAS}

1. Ortega C, Da Costa M, Pereira G. Perfil epidemiológico de la drogadicción en estudiantes universitarios. Rev. Latino-Am. Enfermagem. 2011; 19(May-June):665-672.

2. Campos S, Miranda R. Equipo de Salud de Familia y uso de drogas entre adolescentes. Cogitare Enferm. 2013; 18(3): 482489.

3. Cogollo Z, Arrieta K, Blanco S, Ramos L, Zapato K, Rodríguez Y. Factores psicosociales asociados al consumo de sustancias en estudiantes de una universidad pública. Rev. salud pública. 2011; 13(3): 470-479.
4. OPS. Epidemiología del uso de drogas en América Latina y el Caribe: Un enfoque de salud pública. Disponible en www: http: / /www.paho.org/hq/dmdocuments/2009/epidemiologia _drogas_web.pdf?ua=1 Obtenido en junio de 2016.

5. Quimbayo j, Olivella M. Consumo de marihuana en estudiantes de una universidad colombiana. Rev. salud pública. 2013; 15(1):32-43.

6. Rodríguez R, Pérez M. caracterización clínico epidemiológica de pacientes con adicciones. Consultorio Médico las Mangas. Bayamo 2004-2012. Multimed. Revista médica. Granma. 2014; $18(2): 1-10$

7. González O, Valle D. Estudio de los procesos cognitivos en pacientes adictos a drogas ilícitas.2014. Disponible en: http: / / new.medigraphic.com/cgi-bin/resumen.cgi? IDARTICULO=54516 Revisado en junio 2016.

8. Benitez M, Morós C. prevención del consumo de tóxicos (1.a parte): ¿Qué sustancias consumen nuestros adolescentes? Disponible en www: http://www.fapap.es/files/639-711RUTA/10_PPT_CONSUMO\%20TOXICOS\%200K.pdf Obtenido en junio de 2016.

9. Vinícius M, Soprani D. Miguel de Siqueira M. Uso de alcohol y tabaco entre estudiantes de psicología de la Universidad Federal de Espíritu Santo. J Bras psiquiatr. 2013; 62(1): 22-30.

10. Medrada M, Benítez M. Conceptos básicos sobre drogadicción. Rev Pediatría Aten Primaria. 2006; 8(Supl 3):35-42.

11. Domínguez-Martín A, Miranda-Estribí M., Pedrero-Pérez E., Pérez-López M., Puerta-García C. Estudio de las causas de abandono del tratamiento en un centro de atención de drogodependientes. Trastornos Adictivos. 2008; 10(2): 112-120.

12. Molina M. Evolución histórica del consumo de drogas: concepto, clasificación e implementaciones del consumo prolongado. International e-Journal of Criminal Science. 2008; 2(2):1-30.

13. Fernández J, Touriño R. Evaluación e intervención sobre el trastorno dual en rehabilitación psicosocial. Rehabilitación psicosocial. 2006; 3(1): 9-16.

14. Sánchez E. Tratamientos combinados en la adicción a drogas. Trastornos Adictivos. 2006; 8(1): 42-52.

15. Naciones Unidas. Oficina contra la Droga y el Delito. Abuso de drogas: tratamiento y rehabilitación. Manual sobre tratamiento del abuso de drogas. Guía práctica de planificación y aplicación. Disponible en: www: https://www.unodc.org/ docs/treatment/Guide_S.pdf Obtenido en junio de 2016.

16. Díaz L, Palucci M. El papel de los profesionales en centros de atención en drogas en ambulatorios de la ciudad de Bogotá, Colombia. Rev Latino-Am Enfermagem. 2010; 18:(n.spec): 573-581.

17. Santis R, Hidalgo C, Hayden V, Anselmo E, Rodríguez J, Cartajena F, et al. Consumo de sustancias y conductas de riesgo en consumidores de pasta base de cocaína no consultantes a servicios de rehabilitación. Rev méd Chile. 2007; 135(1):45-53.

18. Ministerio de Salud. Reglamento de centros de Tratamiento y Rehabilitación de personas con consumo perjudicial o dependencia a alcohol y/o drogas. Disponible en: http: / /www.senda.gob.cl/wp-content/uploads/2012/ 08/ReglamentoCentrosdeTratamiento.pdf Obtenido en junio de 2016.

19. Hernández R. La investigación cualitativa a través de entrevistas: su análisis mediante la teoría fundamentada. Cuestiones Pedagógicas. 2014; 23(2013/14):187-210.

20. Valero-Aguayo L, Ortiz-Tallo M, Parra-García M, JimenezGuerra M. Valoración de resultados y perfil psicosocial de un programa de rehabilitación de personas con drogodependencia. Anal. Psicol. 2013; 29(1):38-47.

21. Brito C. Yoga en el tratamiento de adicciones. La experiencia de dos años de práctica de yoga con pacientes del centro de Rehabilitación Takiwasi. Psicoperspectivas. 2010; 9(2): 253-278. 
22. Tomás S. Rehabilitación e integración social en drogodependencias: circuito terapéutico. Salud y drogas. 2007; 7(1):17-22.

23. Córdova J, Rodríguez, Díaz D. Bienestar subjetivo en jóvenes mexicanos usuarios y no usuarios de drogas. Revista Intercontinental de Psicología y Educación. 2010; 12(2): 147-162.

24. Rodríguez S, Mata J, Moreno S. Psicofisiología del ansia por la comida y la bulimia nervosa. Clínica y Salud. 2007; 18(1):99-118.

25. Mené L. Plan de cuidados estandarizado para la alimentación e higiene bucodental en personas drogodependientes en proceso de rehabilitación. Disponible en www: http://invenio2. unizar.es/record/10445/files/TAZ-TFG-2013-069.pdf Obtenido en junio de 2016.

26. Calvo H. Redes de apoyo para la integración social: La familia. Salud y Drogas. 2007; 7(1):45-56.

27. Baptista M, Lemos V, Carneiro A, Morais P. Percepción del apoyo familiar en los consumidores de drogas y su relación con trastornos psiquiátricos. ADICCIONES. 2013; 25(3):220-225.

28. Serrano A, Rodríguez N, Louro I. Afrontamiento familiar a la drogodependencia en adolescentes. Rev Cubana Salud Pública. 2011; 37(2).

29. Albán K, Castillo P. Lineamientos para la implementación de un servicio de alimentación colectiva. Disponible en: http://www.dspace.espol.edu.ec/xmlui/handle/123456789/ 21021 Obtenido en junio de 2016.

30. Caracuel A. Alimentación hospitalaria: del blanco y negro al color. Anales de RACVAO. 2007; 20(1): 43-67.

31. Espinoza L, Rodríguez F, Gálvez J, MacMillan M. Hábitos de alimentación y actividad física en estudiantes universitarios. Rev. chil. nutr. 2011; 38(4):458-465.
32. Franco F, Arboleda L. Aspectos socioculturales y técnicosnutricionales en la alimentación de un grupo de adultos mayores del centro gerontológico Colonia de Belencito de Medellín-Colombia. Perpesct Nutr Humana. 2010; 12(1):61-74.

33. Serrani D. Evaluación neuropsicológica de drogodependientes duales a alcohol y cocaína en período avanzado de abstinencia. Revista Neuropsicológica, Neuropsiquiatra y Neurociencias. 2009; 9(1): 93-113.

34. Esquivel G, Ortiz L, Ortiz R. Mujeres consumidoras de drogas inyectables y sus problemas de salud. Archivos en Medicina Familiar. 2012; 14(2):47-58.

\section{Fuentes de financiamiento}

Este artículo ha sido financiado por los autores.

\section{Conflictos de interés}

Los autores declaran no tener ningún conflicto de interés.

\section{Correspondencia:}

Claudia Troncoso Pantoja

Dirección: Universidad Católica de la Santísima Concepción. Facultad de Medicina

Campus San Andrés. Alonso de Ribera 2850. Concepción. Chile

Teléfono: 56-041-2345427-56-041-2345406

Correo electrónico: ctroncosop@ucsc.cl

Recibido: 18 de marzo de 2016 Aprobado: 28 de junio de 2016 